\title{
DUST IN PLANETARY NEBULAE: OBSERVATIONAL CONSIDERATIONS
}

\author{
Bruce Balick \\ Astronomy Department, University of Washington, Seattle, \\ WA 98195 USA and National Radio Astronomy Observatory, \\ Socorro, NM 87801 USA
}

The question of dust in planetary nebulae dates back a decade to the first then unexpected discoveries of strong infrared (IR) excesses in planetary nebulae (Gillett et al. 1967; Woolf 1969; Gillett and Stein 1969, 1970; Neugebauer and Garmire 1970; Willner et al. 1972). It has been clear from the outset that a substantial fraction, if not most, of the nebular luminosity is somehow converted to IR radiation by the dust. Two important questions are whether, and to what extent, dust influences the ionization structure and chemical properties of the nebula.

In this talk I shall review the recent observational literature which relates to questions concerning the dust. Specifically, I shall explore the amount of dust, its temperature, and briefly reopen questions of its chemical composition. A vast literature is rapidly developing in these areas, and I consider it important to organize the many exciting results here. Some of these results are available only as preprints or talks at this conference; I have had to rewrite parts of this talk twice since the meeting began.

For many years studies of planetary nebulae have concentrated on those which are bright and already well studied. I too will follow the beaten path and emphasize NGC 7027. Nonetheless mention absolutely must be made of the pioneering efforts to extend surveys of planetary nebulae to many previously unstudied objects by Cohen and Barlow (1974). They may yet prove us to be missing the forest for the trees. Their results on the "fossil" planetary nebulae A30 and A78 are very exciting and bear heavily on the question of dust formation. They will be reporting these results later in this meeting.

Of course the best studied of planetary nebulae has been NGC 7027, and we shall later explore whether it is representative of planetary nebulae in general. We have seen its spectrum in great detail already. The total luminosity derived from its spectrum is ( $D$ is the distance)

$$
\mathrm{L}_{\text {tot }} \approx \mathrm{L}_{\mathrm{IR}} \approx 2 \times 10^{4}\left(\frac{\mathrm{D}}{1.8 \mathrm{kpc}}\right)^{2} \mathrm{~L}_{0}
$$


according to Telesco and Harper (1977). While the exciting star of NGC 7027 has never been observed (Miller and Mathews 1972), its temperature has been inferred in a variety of ways. Its temperature, $1.5-2.0 \times 10^{5} \mathrm{~K}$, is consistent with a luminosity $\mathrm{L}_{\star} \approx$ few $\times 10^{4}$ inferred for other exciting stars of about the same temperature from the HarmonSeaton sequence.

At an assumed distance of $1.8 \mathrm{kpc}$, it is clear that since $L_{I R}=$ $L_{\text {dust }} \approx L_{*}$, then one way or another the dust ultimately absorbs ${ }$ virtuallyt all of the stellar energy output. I must point out, however, the reputation of NGC 7027 as being a "dusty" planetary nebula is predicated on $L_{\text {dust }} / L_{*} \sim 1$ whereas if $D$ is less than $1.8 \mathrm{kpc}$, then

$$
\frac{L_{\text {dust }}}{L_{*}} \sim\left(\frac{D}{1.8 \mathrm{kpc}}\right)^{2}<1
$$

Clearly, the question of the distance of NGC 7027 is of considerable relevance to studies of the importance of dust.

Telesco and Harper, using properties for the dust suggested by Aannestad (1975) have estimated the mass of dust to be

$$
\mathrm{M}_{\text {dust }} \sim \operatorname{const}\left(\frac{2 \times 10^{4} \mathrm{~L}_{\odot}}{\mathrm{T}_{\text {dust }}^{4^{+n}}}\right)\left(\frac{\mathrm{D}}{1.8 \mathrm{kpc}}\right)^{2} \mathrm{M}_{\odot} \approx 10^{-3} \mathrm{M}_{\odot}
$$

where the infrared emissivity index $\mathrm{n} \sim 2$. Constants appropriate to graphite yield somewhat larger dust masses, as pointed out by Forrest earlier in the conference. Note the extreme sensitivity of Mast on $\mathrm{T}$ dust; quite obviously cool dust can easily dominate the derived mass. Most earlier estimates of M dust relied on color temperature determinations based on 10-20 fluxes where the flux is dominated by the relatively hot dust; these estimates must be revised upwards. The dust masses of all planetaries should be reevaluated in light of the new long IR wavelength photometry presently underway by Mosley and Harper and reported here by Mosley. Obviously, these results are of tremendous relevance. Moreover, I would like to reemphasize Barlow's comment earlier in which he pointed out that color temperatures derived from broadband fluxes near $10 \mu$ can be overestimated by several tens of degrees if the contribution of line emission is not subtracted.

Theoreticians presently insist that since $L_{\text {dust }} / L_{*} \sim I$, then the dust must be mixed with the ionized gas. The reasoning goes as follows: The dust is heated by the absorption of photons. Inside the nebula most of the photon energy is in the form of stellar radiation, and virtually all of the stellar photons have energies hv>13.6 eV for the highly excited nebulae such as NGC 7027. Outside the nebula the emergent photons consist entirely of nebular emission for which hv<13.6 eV . The absorption cross-section of dust is probably considerably larger at energies above the Lyman limit than below it, so the dust opacity is most likely to exceed unity at higher energies (indeed, the dust opacity 
at $\lambda>0.3 \mu$ must be much less than 1 since we observe planetary nebulae). Hence if a major fraction of the stellar energy is reradiated by dust, then the dust must be directly absorbing stellar radiation and thus the dust must be inside the HII region.

I prefer to adopt an observers' point of view and leave open the question of the location of the dust until it can be measured. A $10 \mu$ map and a $20 \mu$ scan of NGC 7027 were reported by Becklin, Neugebauer, and Wynn-Williams (1973) using spatial resolutions like those of radio synthesis maps (a few arcseconds). The dust which radiates at $\lambda<20 \mu$ and the ionized gas were shown to be spatially coincident; this dust accounts for $1 / 3$ of the infrared luminosity. At longer wavelengths no high spatial resolution information is available. Both Simon and Dyck (1977) using a 15" diaphram and Telesco and Harper (1977) using a 50" diaphram determine the same $\lambda \sim 30 \mu$ flux for NGC 7027 . The nebula fills only $\leqslant 1 / 3$ of the smaller aperture. These results are important because they place the dust within reach of the stellar and/or nebular radiation. On the other hand we heard two results Monday which suggest that the luminous cool dust lies just outside the nebular boundary. These are the molecular hydrogen maps of Beckwith, Becklin, and Neugebauer (reported at this meeting by Dr. Zuckerman) and Dr. Forrests' analysis

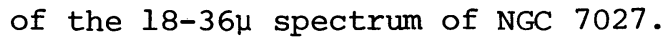

Completely overlooked at the moment is the good possibility that an important fraction of the dust is associated with a faint outer halo of ionized gas seen in many planetaries. The mass of ionized gas in the halo may be comparable to that in the brighter inner nebula. It is conceivable that much of the radiating dust can exist in this halo if the fractional mass of dust and gas are constant. In the case of NGC 7027 an ionized halo must be detected at radio wavelengths. Observations of the required dynamic range have not yet been made.

There are two observations related to the presence of internal extinction of the dust in NGC 7027 which bear on the problems of the dust distribution and its absorption cross-section. The first is the rocket uv spectrum of NGC 7027 obtained by Bohlin, Marionni, and stecher (1975). They conclude from the observed CIV $\lambda 1549$ and CIII] $\lambda 1909$ line intensity ratio that if the interior dust is at all similar to the dust in the interstellar (ISM), then there must be very little absorbing dust in the interior CIV region of the nebula. If, on the other hand, the dust is different than that in the ISM (a possibility for which we shall find some support), then it is possible that at most 0.2 magnitudes of internal absorption exists at $\lambda \sim 0.15 \mu$. As we have seen at this meeting, the CIV to CIII] intensity ratio observed by Bohlin et al. may be entirely explicable by charge-exchange processes, and the question of internal absorption could be entirely superfluous.

On the other hand, an optical study of the [OIII] $\lambda 5007$ line profile shape at various points in the optically bright region of NGC 7027 has been made by Hicks, Phillips, and Reay (1976). Using a Fabry-Perot interferometer, they find that the approaching, or "blue" component of 
the [OIII] line is 30-60\% larger than the "red" component at all points where the line was detected. Assuming that physical conditions are radialily symmetric with respect to the nebular center, they infer an internal extinction $\mathrm{A}_{5007}$ of 0.34 magnitudes. At first sight this result suggests $A 1500$ l assuming, of course, the dust absorbs in the Rayleigh limit; this is inconsistent with the rocket uv results of $\mathrm{A}_{1500} \leq 0.2 \mathrm{mag}$. However, the OIII and CIV regions are not coextensive and, as Dr. Mathis shall discuss, the dust may not be in its Rayleigh limit.

Also of interest is $\mathrm{M}_{\mathrm{dust}} / \mathrm{M}$ gas' the so called dust to gas mass

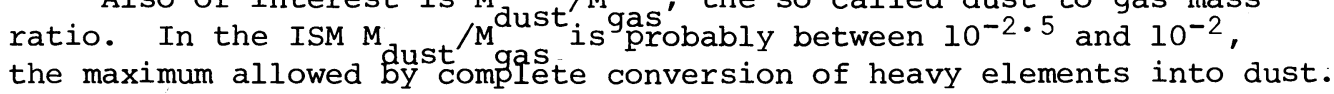
Assuming (and this may be dangerous) that the dust and ionized gas are well mixed in NGC 7027, then

$$
\frac{M_{\text {dust }}}{M_{\text {gas }}} \geqslant \frac{10^{-3}}{0.2}\left(\frac{D}{1.8 \mathrm{kpc}}\right)^{-0.5} \geq 10^{-2.3}
$$

Here, Telesco and Harper's estimate of $M$ dust and an ionized gas mass from radio observations (Webster, Wink, and Altenhoff 1970) are used. At these dust-ionized gas concentrations it is quite possible that dust successfully competes for much of the stellar ionizing radiation. Dr. Mathis will explore this possibility further in the next talk. I must emphasize again, however, that much of the dust may actually lie just beyond the nebular edge or in numerous, small, and neutral condensations within the HII region. If this is the case then dust may be unimportant insofar as the absorption of ionizing radiation is concerned.

While extraordinary in many ways, apparently NGC 7027 is not dissimilar from many other planetary nebulae in terms of its far infrared spectrum and luminosity (Mosley, private communication). Perhaps limited generalizations about the role of dust can be made beyond NGC 7027. However, its $2-13 \mu$ spectrum has been recently explored in detail by Russell, Soifer, and Willner (1977, preprint), and judging from their discussion NGC 7027 is anomalous in many ways. Accurate infrared fluxes and spectra will be obtained for other planetaries in the next few years. Such studies are a very important beginning. However, there is an obvious need for mapping or multiaperture photometry of planetary nebulae at $\lambda \geqslant 30 \mu$ to help settle the questions of the direct source of the dust luminosity and the putative ionization changes attributable to absorption by dust. Additionally, model nebula calculations cannot be trusted to aid in understanding the ionization and thermal structure of planetary nebulae until the absorption properties of the dust as well as improved model atmospheres are available.

Lastly, we briefly consider the dust composition. Monday, Dr. Rank explained the state of our understanding of the nature of the dust and how it is temporarily regressing. I need add little more. It should be emphasized that those IR emission lines of molecular and/or 
lattice transitions of NGC 7027 that are accessible to ground based observations have also been observed in many other types of objects. These sources include young objects as well as highly evolved ones, neutral regions as well as ionized, and an active galactic nucleus (M82) as well as our own galactic disk. These lines, therefore, arise in common, stable, and easily excited transitions of the grains, whatever their composition. Moreover, and this is important, the silicate absorption feature at $10 \mu$ has never been observed in any planetary nebula. This includes NGC 7027 in which heavy foreground extinction is quite obvious. On the other hand, ${ }^{12} \mathrm{CO}$ has been seen towards NGC 7027 by Mufson, Lyon and Marionni (1975). Dr. Zuckerman will address these observations extensively in his talk.

\section{REFERENCES}

Aannestad, P.E. 1975, Ap.J., 200, 30.

Becklin, E.E., Neugebauer, G., and Wynn-Williams, C.G. 1973, Ap.Letters, $15,87$.

Bohlin, R.C., Marionni, P.A., and Stecher, T.P. 1975, Ap.J., 202, 415. Cohen, M. and Barlow, M.J. 1974, Ap.J., 193, 401

Gillett, F.C., Low, F.J., and Stein, W.A. 1967, Ap.J.(Letters), 149, L97. Gillett, F.C. and Stein, W.A. 1969, Ap.J.(Letters), 155, L97. 1970, Ap.J., 159, 817.

Hicks, T.R., Phillips, J.P., and Reay, N.K. 1976, Mon.Not.R.A.S., 176, 409.

Miller, J.S. and Mathews, W.G. 1972, Ap.J., 172,593.

Mufson, S.L., Lyon, J., and Marionni, P.A. 1975, Ap. J. (Letters), 201 L85.

Neugebauer, G. and Garmire, G. 1970, Ap.J.(Letters), 161, L91.

Russell, R.W., Soifer, B.T., and Willner, S.P. 1977 (preprint).

Simon, T. and Dyck, H.M. 1977 (to be published)

Telesco, C.M., and Harper, D.A. 1977, Ap.J., 211, 475.

Webster, W.J., Wink, J.E., and Altenhoff, W.J. 1970, Ap.Letters, 7, 47. Willner, S.I., Becklin, E.E., and Visvanathan, N. 1972, Ap.J., 177̃, 699. Woolf, N.J. 1969, Ap.J. (Letters), 157, L37. 